\title{
The correction of hadronic nucleus polarizability to hyperfine structure of light muonic atoms
}

\author{
A.V. Eskin ${ }^{1, \star}$, A.P. Martynenko ${ }^{1, \star \star}$, and E.N. Elekina ${ }^{2}$ \\ ${ }^{1}$ Samara U., 443086, Samara, Moskovskoe shosse 34 \\ ${ }^{2}$ Samara State U. of Architecture and Civil Engineering, 443001, Samara, Molodogvardeiskaya Str. 194
}

\begin{abstract}
The calculation of hadronic polarizability contribution of the nucleus to hyperfine structure of muonic hydrogen and helium is carried out within the unitary isobar model and experimental data on the polarized structure functions of deep inelastic lepton-proton and lepton-deuteron scattering. The calculation of virtual absorption cross sections of transversely and longitudinally polarized photons by nucleons in the resonance region is performed in the framework of the program MAID.
\end{abstract}

\section{Introduction}

Precise investigation of the Lamb shift and hyperfine structure of light muonic atoms is a fundamental problem for testing the Standard model and establishing the exact values of its parameters, as well as searching for effects of new physics. At present, the relevance of these studies is primarily related to experiments conducted by the collaboration CREMA (Charge Radius Experiments with Muonic Atoms) [1-4] with muonic hydrogen and deuterium by methods of laser spectroscopy. So, as a result of measuring the transition frequency $2 P_{3 / 2}^{F=2}-2 S_{1 / 2}^{F=1}$ a more accurate value of the proton charge radius was found to be $r_{E}=0.84087(39) \mathrm{fm}$, which is different from the value recommended by CODATA for $7 \sigma$ [5]. The CODATA value is based on the spectroscopy of the electronic hydrogen atom and on electron-nucleon scattering. The measurement of the transition frequency $2 P_{3 / 2}^{F=1}-2 S_{1 / 2}^{F=0}$ for the singlet $2 S$ of the state $(\mu p)$ allowed to obtain the hyperfine splitting of the $2 S$ energy level in muonic hydrogen, and also the values of the Zemach's radius $r_{Z}=1.082(37) \mathrm{fm}$ and magnetic radius $r_{M}=0.87(6) \mathrm{fm}$. The first measurement of three transition frequencies between energy levels $2 P$ and $2 S$ for muonic deuterium $\left(2 S_{1 / 2}^{F=3 / 2}-2 P_{3 / 2}^{F=5 / 2}\right),\left(2 S_{1 / 2}^{F=1 / 2}-2 P_{3 / 2}^{F=3 / 2}\right),\left(2 S_{1 / 2}^{F=1 / 2}-2 P_{3 / 2}^{F=1 / 2}\right)$ allowed to obtain in 2.7 times the more accurate value of the charge radius of the deuteron, which is also less than the value recommended by CODATA [5], by $7.5 \sigma$ [4]. As a result, a situation emerges when there is an inexplicable discrepancy between the values of such fundamental parameters, like the charge radius of a proton and deuteron, obtained from electronic and muonic atoms. In the process of searching for possible solutions of the proton charge radius "puzzle" various hypotheses were formulated, including the idea of the nonuniversality of the interaction of electrons and muons with nucleons. Preliminary experimental data for muonic helium ions show that there is no large discrepancy in obtained charge radii in comparison with CODATA.

\footnotetext{
$\star^{\star}$ e-mail: eskin33@mail.ru

${ }^{\star}$ e-mail: a.p.martynenko@samsu.ru
} 
In the experiments of the CREMA collaboration one very important task is solved: to obtain an order of magnitude more accurate values of the charge radii of the simplest nuclei (proton, deuteron, helion, alpha particle ....) that enter into one form or another into theoretical expressions for intervals of fine or hyperfine structure of the spectrum. In this case, high sensitivity of characteristics of the bound muon to distribution of charge density and magnetic moment of the nucleus is used. Successful realization of this program is possible only in combination with precise theoretical calculations of various energy intervals, measured experimentally. In this way, the problem of a more accurate theoretical construction of the particle interaction operator in quantum electrodynamics, the calculation of new corrections in the energy spectrum of muonic atoms acquires a special urgency [6-8]. The aim of this work consists in the calculation of the deuteron, helion and triton polarizability correction to the hyperfine splitting (HFS). We perform a calculation of hadronic polarizability contribution using the isobar model describing the processes of photo- and electroproduction of $\pi, \eta$ mesons, nucleon resonances on the nucleon in the resonance region, and experimental data on the nucleon and deuteron polarized structure functions obtained in non-resonance region.

\section{General formalism}

The leading order polarizability contribution to HFS is determined by two-photon exchange diagrams, shown in Fig. 1. The corresponding amplitudes of virtual Compton scattering on the nucleus can be represented as a convolution of antisymmetric parts of the lepton and hadron tensors which have the following form $[9,10]$ :

$$
\begin{gathered}
L_{\mu \nu}^{A}=\frac{1}{4} \operatorname{Tr}\left\{\left(1+\gamma^{0}\right) \gamma_{5} \hat{s}_{1}\left[\gamma_{1}^{\mu} \frac{\hat{p}_{1}+\hat{k}+m_{1}}{\left(p_{1}+k\right)^{2}-m_{1}^{2}} \gamma_{1}^{v}+\gamma_{1}^{v} \frac{\hat{p}_{1}-\hat{k}+m_{1}}{\left(p_{1}-k\right)^{2}-m_{1}^{2}} \gamma_{1}^{\mu}\right]\right\}, \\
W_{\mu \nu}^{A}=i \epsilon_{\mu \nu \alpha \beta} k^{\alpha}\left\{s_{2}^{\beta} \frac{H_{1}\left(\nu, Q^{2}\right)}{\left(p_{2} \cdot k\right)}+\frac{\left[\left(p_{2} k\right) s_{2}^{\beta}-\left(s_{2} k\right) p_{2}^{\beta}\right]}{\left(p_{2} \cdot k\right)^{2}} H_{2}\left(\nu, Q^{2}\right)\right\},
\end{gathered}
$$

where $m_{1}, m_{2}$ are the lepton and nucleus masses, the nucleus four-momentum $p_{2}=\left(m_{2}, 0\right), \epsilon_{\mu v \alpha \beta}$ is the totally antisymmetric tensor in four dimensions. $s_{1}, s_{2}$ are spin four vectors of the lepton and nucleus. $H_{1}, H_{2}$ are the structure functions of polarized scattering. The invariant quantity $p_{2} \cdot k$ is related to the energy transfer $v$ in the nucleus rest frame: $p_{2} \cdot k=m_{2} v$. The invariant mass of the electroproduced hadronic system $W$ is then $W^{2}=m_{2}^{2}+2 m_{2} v-Q^{2}=m_{2}^{2}+Q^{2}(1 / x-1)$. After computing the convolution of two tensors (1) and (2), we obtain:

$$
L_{\mu \nu}^{A} W_{\mu \nu}^{A}=\frac{4}{3}\left(\mathbf{s}_{1} \mathbf{s}_{2}\right) \frac{m_{2} k^{2}}{k^{4}-4 m_{1}^{2} k_{0}^{2}}\left[\left(k_{0}^{2}+2 k^{2}\right) \frac{H_{1}}{\left(p_{2} \cdot k\right)}+3 k_{0}^{2} k^{2} \frac{H_{2}}{\left(p_{2} \cdot k\right)^{2}}\right] .
$$

According to the optical theorem the imaginary part of the forward Compton amplitude is related to the cross section of inelastic scattering of off-shell photons from protons: $\operatorname{ImH}_{1}\left(v, Q^{2}\right)=g_{1}\left(v, Q^{2}\right) / v$, $\operatorname{ImH}_{2}\left(v, Q^{2}\right)=m_{2} g_{2}\left(v, Q^{2}\right) / v^{2}$. As a result, neglecting the lepton mass the nucleus polarizability contribution to HFS can be presented in the form [11-14]:

$$
\begin{aligned}
& \Delta E_{p o l}^{h f s}=\frac{Z \alpha m_{1}}{2 \pi m_{2} \mu_{N}} E_{F}\left(\Delta_{1}+\Delta_{2}\right)=\left(\delta_{1}^{p}+\delta_{2}^{p}\right) E_{F}=\delta_{p o l} E_{F}, \\
& \Delta_{1}=\int_{0}^{\infty} \frac{d Q^{2}}{Q^{2}}\left\{\frac{9}{4} F_{2}^{2}\left(Q^{2}\right)-4 m_{2} \int_{v_{t h}}^{\infty} \frac{d v}{v^{2}} \beta_{1}(\theta) g_{1}\left(v, Q^{2}\right)\right\},
\end{aligned}
$$




$$
\Delta_{2}=-12 m_{2} \int_{0}^{\infty} \frac{d Q^{2}}{Q^{2}} \int_{v_{t h}}^{\infty} \frac{d v}{v^{2}} \beta_{2}(\theta) g_{2}\left(v, Q^{2}\right),
$$

where $v_{t h}$ determines the pion-nucleus threshold:

$$
v_{t h}=m_{\pi}+\frac{m_{\pi}^{2}+Q^{2}}{2 m_{2}},
$$

and the functions $\beta_{1,2}$ have the form:

$$
\begin{gathered}
\beta_{1}(\theta)=3 \theta-2 \theta^{2}-2(2-\theta) \sqrt{\theta(\theta+1)}, \\
\beta_{2}(\theta)=1+2 \theta-2 \sqrt{\theta(\theta+1)}, \theta=v^{2} / Q^{2} .
\end{gathered}
$$

$F_{2}\left(Q^{2}\right)$ is the Pauli form factor of the nucleus. The dependence on the mass of the lepton in (5)-(6) can also be taken into account, which leads to a certain modification of the functions $\beta_{i}\left(v, Q^{2}\right)[10,14]$. This is important for increasing the accuracy of calculations in the case of muonic atoms.

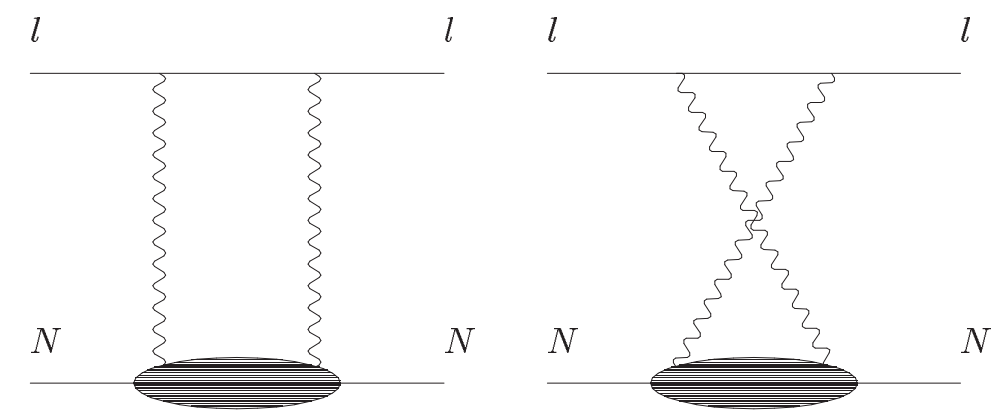

Figure 1. Two-photon Feynman amplitudes determining the correction of the nucleus polarizability to the hyperfine splitting of muonic atom.

Having information on the polarization structure functions of the nuclei, one can integrate into (4) and obtain this correction. The nucleus spin-dependent structure functions $g_{1}\left(v, Q^{2}\right), g_{2}\left(v, Q^{2}\right)$ can be measured in the inelastic scattering of polarized leptons on polarized nuclei. Accurate measurements of proton and deuteron polarized structure functions were made in SLAC, CERN and DESY [1520]. These experimental data can be used for the calculation the contribution in the nonresonance region where the invariant mass $W$ must be greater than the mass of any resonance $N^{*}$ in the reaction $\gamma^{*}+N \rightarrow N^{*}$. The threshold between the resonance region and the deep-inelastic region is not well defined, but it is usually taken to be at about $W^{2}=4 \mathrm{GeV}^{2}$. On the other hand in the resonance region we need theoretical model describing polarized structure functions $g_{1,2}\left(v, Q^{2}\right)$ since experimental data in this region are clearly insufficient. First of all, we can express functions $g_{1,2}\left(v, Q^{2}\right)$ in terms of virtual photon absorption cross sections as follows:

$$
\begin{aligned}
& g_{1}\left(v, Q^{2}\right)=\frac{m_{2} \cdot K}{8 \pi^{2} \alpha\left(1+Q^{2} / v^{2}\right)}\left[\sigma_{1 / 2}^{T}\left(v, Q^{2}\right)-\sigma_{3 / 2}^{T}\left(v, Q^{2}\right)+\frac{2 \sqrt{Q^{2}}}{v} \sigma_{1 / 2}^{T L}\left(v, Q^{2}\right)\right], \\
& g_{2}\left(v, Q^{2}\right)=\frac{m_{2} \cdot K}{8 \pi^{2} \alpha\left(1+Q^{2} / v^{2}\right)}\left[-\sigma_{1 / 2}^{T}\left(v, Q^{2}\right)+\sigma_{3 / 2}^{T}\left(v, Q^{2}\right)+\frac{2 v}{\sqrt{Q^{2}}} \sigma_{1 / 2}^{T L}\left(v, Q^{2}\right)\right],
\end{aligned}
$$


where $K$ is the kinematical flux factor for virtual photons. The virtual photon absorption cross sections have superscripts referring to the initial and final photon polarization being longitudinal $\mathrm{L}$ or transverse T. The superscript TL is for the case when the photon polarization direction changes during the interaction. The subscripts refer to the total spin of the photon-nucleus system.

Let us briefly describe the basic formulas that underlie the numerical results. To construct the polarized structure functions (10)-(11) in resonance region we use the Breit-Wigner parameterization for the photoabsorption cross sections [21-28]. In the considered region of the variables $k^{2}, W$ the most important contribution is given by five resonances: $P_{33}(1232), S_{11}(1535), D_{13}(1520), P_{11}(1440)$, $F_{15}(1680)$. Accounting for the resonance decays to the $N \pi-$ and $N \eta-$ states we can express the absorption cross sections $\sigma_{1 / 2}^{T}$ and $\sigma_{3 / 2}^{T}$ as follows:

$$
\sigma_{1 / 2,3 / 2}^{T}=\left(\frac{k_{R}}{k}\right)^{2} \frac{W^{2} \Gamma_{\gamma} \Gamma_{R \rightarrow N \pi}}{\left(W^{2}-M_{R}^{2}\right)^{2}+W^{2} \Gamma_{\text {tot }}^{2}} \frac{4 m_{N}}{M_{R} \Gamma_{R}}\left|A_{1 / 2,3 / 2}\right|^{2},
$$

where $A_{1 / 2,3 / 2}$ are transverse electromagnetic helicity amplitudes,

$$
\Gamma_{\gamma}=\Gamma_{R}\left(\frac{k}{k_{R}}\right)^{j_{1}}\left(\frac{k_{R}^{2}+X^{2}}{k^{2}+X^{2}}\right)^{j_{2}}, X=0.3 \mathrm{GeV} .
$$

The resonance parameters $\Gamma_{R}, M_{R}, j_{1}, j_{2}, \Gamma_{t o t}$ are taken from [23, 24, 29]. In accordance with [23, 25] the parameterization of one-pion decay width is

$$
\Gamma_{R \rightarrow N \pi}(q)=\Gamma_{R} \frac{M_{R}}{M}\left(\frac{q}{q_{R}}\right)^{3}\left(\frac{q_{R}^{2}+C^{2}}{q^{2}+C^{2}}\right)^{2}, C=0.3 \mathrm{GeV}
$$

for the $P_{33}(1232)$ and

$$
\Gamma_{R \rightarrow N \pi}(q)=\Gamma_{R}\left(\frac{q}{q_{R}}\right)^{2 l+1}\left(\frac{q_{R}^{2}+\delta^{2}}{q^{2}+\delta^{2}}\right)^{l+1},
$$

for resonances $D_{13}(1520), P_{11}(1440), F_{15}(1680) . l$ is the pion angular momentum and $\delta^{2}=\left(M_{R}-\right.$ $\left.m_{N}-m_{\pi}\right)^{2}+\Gamma_{R}^{2} / 4$. Here $q(k)$ and $q_{R}\left(k_{R}\right)$ denote the c.m.s. pion (photon) momenta of resonances with the mass $M$ and $M_{R}$ respectively. In the case of $S_{11}(1535)$ we take into account $\pi N$ and $\eta N$ decay modes [25]:

$$
\Gamma_{R \rightarrow \pi, \eta}=\frac{q_{\pi, \eta}}{q} b_{\pi, \eta} \Gamma_{R} \frac{q_{\pi \eta}^{2}+C_{\pi, \eta}^{2}}{q^{2}+C_{\pi, \eta}^{2}},
$$

where $b_{\pi, \eta}$ is the $\pi(\eta)$ branching ratio.

The cross section $\sigma_{1 / 2}^{T L}$ is determined by an expression similar to (12), containing the product $\left(S_{1 / 2}^{*} \cdot A_{1 / 2}+A_{1 / 2}^{*} S_{1 / 2}\right)$ [15]. The calculation of helicity amplitudes $A_{1 / 2}, A_{3 / 2}$ and longitudinal amplitude $S_{1 / 2}$, as functions of $Q^{2}$, was done on the basis of constituent quark model (CQM) in [30-35]. The program of numerical calculation of cross sections (12) was successfully realized by the authors of $[36,37]$ within the unitary isobar model framework known as the MAID package (http://www.kphuni-mainz.de/MAID). In the unitary isobar model $[36,37]$ accounting for the Born terms, the vector meson, nucleon resonance contributions and interference terms we calculate the cross sections $\sigma_{1 / 2,3 / 2}^{T}, \sigma_{1 / 2}^{T L}$ by means of numerical program MAID in the resonance region as the functions of two variables $W$ and $Q^{2}$. The obtained nucleon polarized structure functions $g_{1,2}\left(W, Q^{2}\right)$ are then used for a construction of nucleus structure functions and calculation the polarizability contribution. In Figs. 2,3 we show the obtained structure functions $g_{1,2}\left(W, Q^{2}\right)$. 


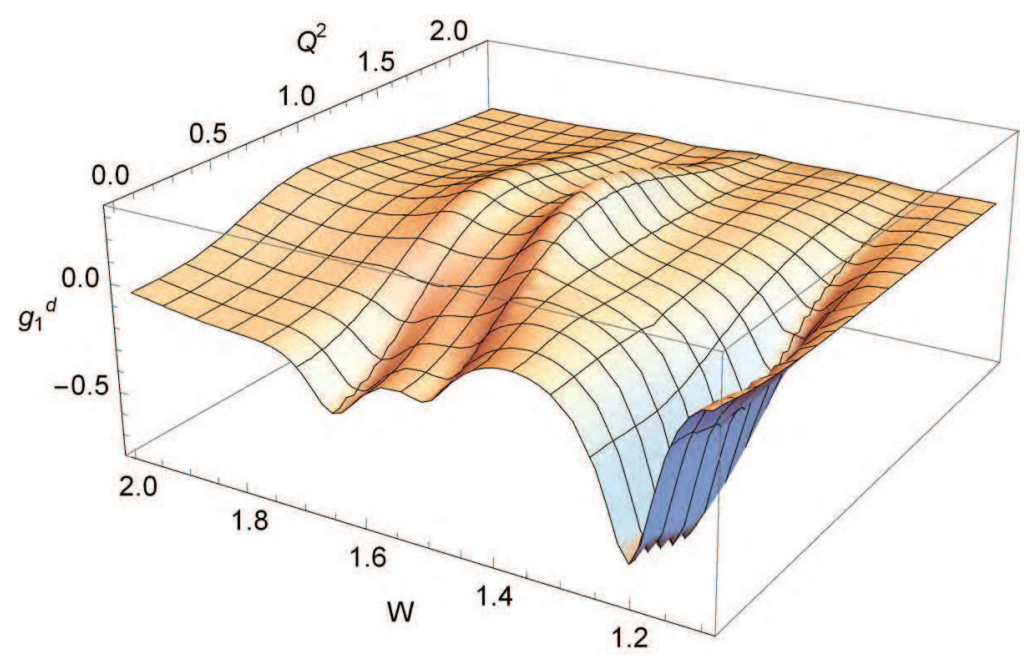

Figure 2. The deuteron polarized structure function $g_{1}^{d}\left(W, Q^{2}\right)$ as function of $Q^{2}\left(0 \div 2.0 . \mathrm{GeV}^{2}\right.$ and $\mathrm{W}(1.1 \div$ 2.0) $\mathrm{GeV}$.

Our calculation of polarizability contribution in deep inelastic region is based on experimental data from [15-20]. As was shown in previous paper [10] nucleon polarized structure functions can be expressed through polarized quark and gluon distributions which obey to the evolution equations $[38,39]$. Solving $Q^{2}$ evolution equations we can construct nucleon functions $g_{1}\left(v, Q^{2}\right), g_{2}\left(v, Q^{2}\right)$ which agree well with experimental data and the following parameterization [15-20, 40]:

$$
g_{1}^{p, d}\left(x, Q^{2}\right)=a_{1} x^{a_{2}}\left(1+a_{3} x+a_{4} x^{2}\right)\left[1+a_{5} f\left(Q^{2}\right)\right] F_{1}^{p, d}\left(x, Q^{2}\right),
$$

where the superscript index $\mathrm{p}, \mathrm{d}$ corresponds to the proton or deuteron. Numerical integration is performed with $f\left(Q^{2}\right)=-\ln Q^{2}$ corresponding to the perturbative QCD behaviour. The calculation of the second part of the correction $\delta_{\text {pol }}$ in (4) in nonresonance region is carried out by means of Wandzura-Wilchek relation as in [10].

\section{Numerical results}

Most part of numerical calculation is devoted to muonic deuterium. To the hadronic contribution we include contributions that are determined by the nuclear reactions of the production of $\pi-, \eta$, and $K$ mesons on nucleons, the production of nucleon resonances. In the approximation, which is then used for the calculation the deuteron appears as a loosely coupled system of the proton and neutron, so the deuteron polarized structure function can be presented as a sum of the proton and neutron structure functions:

$$
g_{i}^{d}\left(W, Q^{2}\right)=g_{i}^{p}\left(W, Q^{2}\right)+g_{i}^{n}\left(W, Q^{2}\right) .
$$

The MAID program allows us to calculate separately the proton and neutron structure functions $g_{i}^{p, n}\left(W, Q^{2}\right)$.

For the integration in (4) an important role is played by the Gerasimov-Drell-Hern (GDH) sum rule [41-45] which connects an energy-weighted integral of the difference of the helicity dependent 


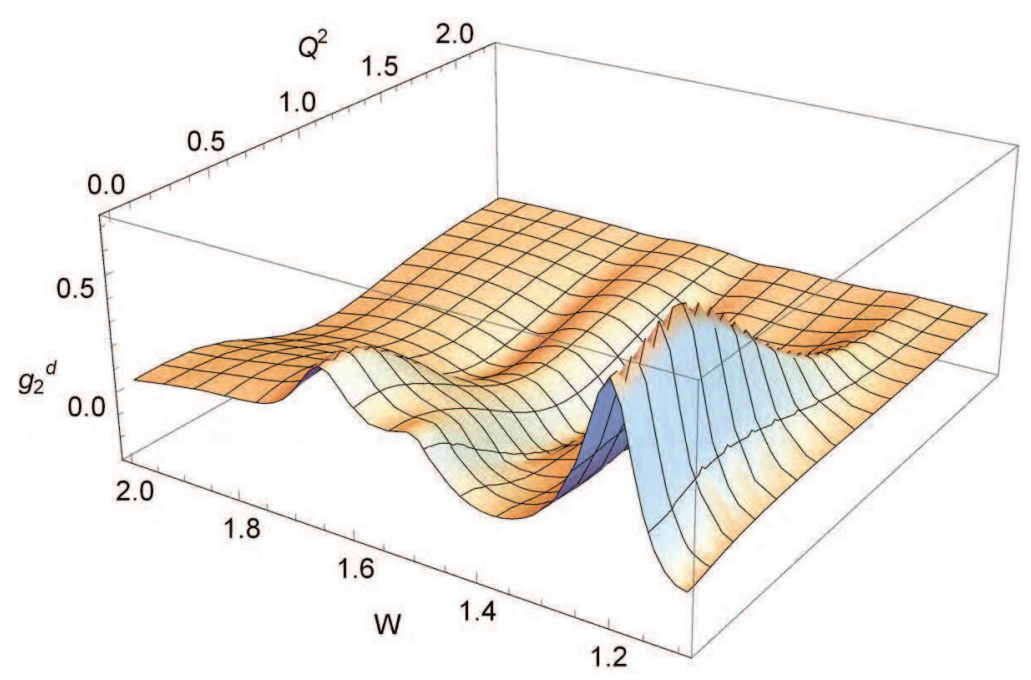

Figure 3. The deuteron polarized structure function $g_{2}^{d}\left(W, Q^{2}\right)$ as function of $Q^{2}\left(0 \div 2.0 . \mathrm{GeV}^{2}\right.$ and $\mathrm{W}(1.1 \div$ 2.0) $\mathrm{GeV}$.

real-photon absorption cross sections with the anomalous contribution $\kappa=\frac{\mu_{N} m_{2}}{e s_{2}}-Z$ to the magnetic moment $\mu$ of the nucleus:

$$
I_{G D H}=\int_{v_{t h}}^{\infty} \frac{\sigma_{p}-\sigma_{a}}{v} d v=4 \pi^{2} \kappa^{2} \frac{\alpha s_{2}}{m_{2}^{2}},
$$

where $v$ is the photon energy, $\sigma_{p}$ and $\sigma_{a}$ are the total photoabsorption cross sections for parallel and antiparallel orientation of photon and nucleus spins, respectively. The lower limit of the integral, $v_{t h}$, corresponds to pion production and photodisintegration threshold for a nucleonic and nuclear target, respectively. Strictly speaking, in order for the integral in (4) to converge over the variable $Q$, the sum rule (19) must exactly be satisfied. The GDH sum rule is satisfied for any nucleus, including a proton and a neutron. Since the deuteron structure function satisfies (18), it is necessary to achieve the sum rules for the proton and neutron. Since the neutron and the proton have large anomalous magnetic moments $\left.\kappa_{p}=1.79, \kappa_{n}=-1.91\right)$, we obtain large values for the integral $I_{G D H}^{p, n}(19)$. In turn, in the case of a deuteron, a small value of $\operatorname{AMM}\left(\kappa_{d}=-0.143\right)$ leads to a small value $I_{G D H}^{d}=-0.65 \mu b$ (19), which is two orders of magnitude smaller than the corresponding values for nucleons. When the deuteron is represented in the form of a state of two almost free nucleons, we find that the quantity in right part of (19) is equal to $437 \mu b$. There are also other channels ( $\gamma d \rightarrow p n$ ) which can not be treated in the quasi-free approximation but which contribute to (19). An analysis carried out in [46, 47] showed that this value decreases significantly when taking into account the photodisintegration channel, which is not considered in this paper. Therefore, representing the deuteron in the form of the sum of a proton and a neutron, we add in (4) two terms with the Pauli form factors of the proton and the neutron to ensure the fulfilment of the sum rule and the subsequent integration into (4). Calculations in the MAID show that the sum rule for a proton is satisfied with a sufficiently high accuracy, whereas for a neutron the difference between the left and right parts in (19) reaches $30 \%$ (see Table 1).

To avoid this difficulty and obtain however an estimate of hadronic contribution to the neutron and deuteron polarizability we introduce a cutoff of the momentum integral over Q in (4) at some value $\kappa \approx 0.01 \mathrm{GeV}$, supposing that the GDH sum rule for the neutron holds exactly and the region of small 
Table 1. The contributions to the GDH sum rule for the proton and neutron.

\begin{tabular}{|c|c|c|}
\hline Contribution to GDH integral & $I_{G D H}^{p}, \mu b$ & $I_{G D H}^{n}, \mu b$ \\
\hline Contribution of $N \pi$ states & 165.9 & 133.2 \\
\hline Contribution of $N \eta$ states & -8.9 & -5.7 \\
\hline Contribution of K-mesons & -1.8 & -3.0 \\
\hline Contribution of $N \pi \pi$ states & 47.8 & 50.5 \\
\hline Total contribution & 203.0 & 175.0 \\
\hline The GDH value & 204.8 & 232.5 \\
\hline
\end{tabular}

$\mathrm{Q}(0 \div 0.01) \mathrm{GeV}$ does not give essential contribution to general value of correction (4). Similar cutoff procedure at small values $\mathrm{Q}$ was used in [48] with $\kappa \approx 0.045 \mathrm{GeV}$, in which different corrections to deuterium hyperfine structure were considered from the two-photon exchange amplitudes. As a result total value of the polarizability correction for muonic deuterium including the resonance and nonresonance regions is equal to $0.13 \mathrm{meV}$. In the case of He- 3 total polarizability contribution 0.06 $\mathrm{meV}$ is determined by unpaired neutron because two protons have opposite spins and do not contribute to hyperfine splitting. The similar situation occurs for the triton in which two neutrons form closed shell. In muonic tritium the polarizability correction is equal to $0.05 \mathrm{meV}$. Total error of our calculation is estimated in $30 \%$ which is determined mainly by the uncertainty from two-pion contribution and the cutoff procedure used above. The obtained values of polarizability corrections should be used for obtaining total values of hyperfine splitting in light muonic atoms [6, 49-51].

\section{References}

[1] R. Pohl, A. Antognini, F. Nez et al., Nature 466, 213 (2010).

[2] A. Antognini et al., Science 339, 417 (2013).

[3] A. Antognini et al., Ann. Phys. (NY) 331, 127 (2013).

[4] R. Pohl, F. Nez, L. M. P. Fernandes et al., Science 353, 669 (2016).

[5] P. J. Mohr, D. B. Newell, and B. N. Taylor (CODATA Recommended Values of the Fundamental Physical Constants: 2014) Rev. Mod. Phys. 88, 035009 (2016).

[6] A. P. Martynenko, F. A. Martynenko, R. N. Faustov, J. Exp. Theor. Phys. 124, 895 (2017).

[7] A. V. Eskin, A. P. Martynenko, E. N. Elekina, EPJ Web Conf. 132, 03055 (2017).

[8] R. N. Faustov, A. P. Martynenko, G. A. Martynenko, V. V. Sorokin, Phys. Lett. B 733, 354 (2014). 
[9] G. M. Zinov'ev, B. V. Struminsky, R. N. Faustov, V. L. Chernyak, Sov. J. Nucl. Phys. 11, 715 (1970).

[10] E. V. Cherednikova, R. N. Faustov, A. P. Martynenko, Nucl. Phys. A 703, 365 (2002).

[11] R. N. Faustov and A. P. Martynenko, Eur. Phys. J. C 24, 281 (2002).

[12] R. N. Faustov and A. P. Martynenko, Phys. Atom. Nucl. 65, 265 (2002).

[13] C. E. Carlson, Can. J. Phys. 85, 429 (2007).

[14] C. E. Carlson, V. Nazaryan, K. Griffioen, Phys. Rev. A 83, 042509 (2011).

[15] K. Abe, T. Akagi, P. L. Anthony et al., Phys. Rev. D 58, 112003 (1998).

[16] K. Abe et al., Phys. Rev. Lett. 78, 815 (1997).

[17] P. L. Anthony et al., Phys. Lett. B 458, 529 (1999).

[18] G. S. Mitchell, Preprint SLAC-PUB-8104, 1999.

[19] D. Adams et al., Phys. Rev. D 56, 5330 (1997).

[20] D. Adeva et al., Phys. Rev. D 60, 072004 (1999).

[21] R. L. Walker, Phys. Rev. 182, 1729 (1969).

[22] R. A. Arndt, R. L. Workman, Z. Li et al., Phys. Rev. C 42, 1864 (1990).

[23] S. Teis, W. Cassing, M. Effenberger et al., Z. Phys. A 356, 421 (1997).

[24] M. Effenberger, A. Hombach, S. Teis et al., Nucl. Phys. A 613, 353 (1997).

[25] B. Krusche, J. Ahrens, G. Anton et al., Phys. Rev. Lett. 74, 3736 (1995).

[26] N. Bianchi, V. Muccifora, E. Sanctis et al., Phys. Rev. C 54, 1688 (1996).

[27] D. Drechsel, O. Hanstein, S. S. Kamalov et al., Nucl. Phys. A 645, 145 (1999).

[28] Y.-B. Dong, Eur. Phys. Jour. A 1, 347 (1998).

[29] C. Patrignani et al. (Particle Data Group), Chin. Phys. C 40, 100001 (2016).

[30] Z. Li and Y.-B. Dong, Phys. Rev. D 54, 4301 (1996).

[31] R. Koniuk and N. Isgur, Phys. Rev. D 21, 1888 (1980).

[32] F. E. Close, Z. Li, Phys. Rev. D 42, 2207 (1990).

[33] S. Capstick, Phys. Rev. D 46, 2864 (1992).

[34] Zhenping Li, V. Burkert, Zhujun Li, Phys. Rev. D 46, 70 (1992).

[35] M. Warns, W. Pfeil, H. Rollnik, Phys. Rev. D 42, 2215 (1990).

[36] W.-T. Chiang, S. N. Yang, L. Tiator, D. Drechsel, Nucl. Phys. A 700, 429 (2002).

[37] D. Drechsel, S. S. Kamalov, G. Krein, L. Tiator, Phys. Rev. D 59, 094021 (1999).

[38] G. Altarelli, R. D. Ball, S. Forte et al. Nucl. Phys. B 496, 337 (1997).

[39] M. Hirai, S. Kumano, M. Miyama, Comp. Phys. Comm. 108, 38 (1998).

[40] R. D. Erbacher, SLAC-Report-546 (1999).

[41] S. B. Gerasimov, Sov. J. Nucl. Phys. 2, 430 (1966).

[42] S. D. Drell and A. C. Hern, Phys. Rev. Lett. 16, 908 (1966).

[43] D. Drechsel and G. Krein, Phys. Rev. D 58, 116009 (1998).

[44] S. Costanza, J. of Phys.: Conf. Ser. 349, 012011 (2012).

[45] A. Airapetian et al. [The HERMES Collaboration], Preprint DESY 02-172, (2002).

[46] H. Arenhövel, G. Kreß, R. Schmidt, P. Wilhelm, Phys. Lett. B 407, 1 (1997).

[47] H. Arenhövel, Phys. Lett. B 595, 223 (2004).

[48] I.B. Khriplovich and A.I. Milstein, J. Theor. Exp. Phys. 125, 205 (2004).

[49] R. N. Faustov and A. P. Martynenko, J. Theor. Exp. Phys. 98, 39 (2004).

[50] A. P. Martynenko, J. Exp. Theor. Phys. 106, 691 (2008).

[51] F. A. Martynenko, R. N. Faustov, and A. P. Martynenko, EPJ WoC 132, 03032 (2017). 